\title{
DOR MUSCULAR E ATIVIDADE DE CREATINA QUINASE APÓS AÇÕES EXCÊNTRICAS: UMA ANÁLISE DE CLUSTER
}

\author{
MUSCLE SORENESS AND CREATINE KINASE ACTIVITY AFTER ECCENTRIC ACTIONS: \\ A CLUSTER ANALYSIS
}

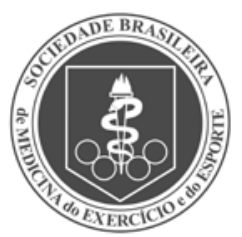

Artigo Original

\author{
DOLOR MUSCULAR Y ACTIVIDAD DE CREATINA KINASA TRAS ACCIONES EXCÉNTRICAS: \\ UN ANÁLISIS DE CLUSTER
}

Felipe Romano Damas Nogueira' (Educador Físico)

Mara Patricia Traina Chacon-Mikahil (Educadora Física)

Felipe Cassaro Vechin'

(Educador Físico)

Ricardo Paes de Barros Berton'

(Educador Físico)

Claudia Regina Cavaglieri ${ }^{1}$

(Bioquímica)

Cleiton Augusto Libardi ${ }^{1}$

(Educador Físico)

1. Universidade Estadual de Campinas (Unicamp), Campinas, SP, Brasil.

\section{Correspondência:}

Laboratório Fisiologia do Exercício, Faculdade de Educação Física da Unicamp. Av. Erico Veríssimo, 701, Cidade Universitária, Campinas, SP, Brasil 13083-851.

felipedamasfl@gmail.com

\section{RESUMO}

Introdução: O exercício excêntrico máximo promove queda da capacidade de geração de força, aumento de dor muscular de início tardio (DMIT) e extravasamento de creatina quinase (CK) no sangue. No entanto, essas respostas ao exercício excêntrico demonstram alta variabilidade interindivíduos. Objetivos: Classificar e analisar essas respostas segundo a vulnerabilidade individual, por meio da magnitude da redução dos níveis de força após exercício excêntrico. Métodos: Dezoito sujeitos ( 11 homens e sete mulheres com idade entre 18 e 71 anos) saudáveis realizaram cinco séries de seis ações excêntricas máximas para flexores do cotovelo em dinamômetro isocinético $\left(90^{\circ} \mathrm{s}^{-1}\right.$, amplitude de movimento de 80 a $\left.20^{\circ}\right)$. Foram analisadas contração voluntária muscular concêntrica máxima (CVM), DMIT e CK pré, pós (exceto CK), 24 h, 48 h, 72 h e 96 h após o protocolo. Os indivíduos foram classificados como "baixos" (BR) e "altos" respondedores (AR) ao pico de queda de CVM pós-exercício seguindo-se uma análise de cluster e comparação entre grupos para CVM, DMIT e CK com teste- $t$ independente. Resultados: A comparação entre BR e AR indicou maiores valores de queda de CVM para o grupo AR (AR: - $30,7 \pm 3,3 \%$; BR: $-14,1 \pm 2,2 \%, p<0,001$ ) e maior DMIT também para o grupo AR (AR: $33,0 \pm 8,6 \mathrm{~mm} ; \mathrm{BR}: 10,2 \pm 2,5 \mathrm{~mm}, p=0,033$ ); no entanto, os valores de pico de atividade de CK não diferiram entre os grupos (AR: 232,1 $\pm 54,8$ UI.L-1 ; BR: 306,1 $\pm 82,3$ UI.L-1 $, P=0,490$ ). Conclusão: A classificação dos indivíduos em grupos de baixos e altos respondedores foi importante para demonstrar que o grupo mais responsivo à queda de força após exercício excêntrico também evidencia maiores níveis de DMIT; contudo, para a CK essa relação não pôde ser estabelecida.

Palavras-chave: exercício, força muscular, músculo esquelético, treinamento de resistência.

\section{ABSTRACT}

Introduction: Maximal eccentric exercise promotes decreases in muscular strength, increases in delayed-onset muscle soreness (DMIT) and leakage of creatine kinase (CK) in the blood. However, these responses to eccentric exercise demonstrated large inter-individual variability. Objectives: To classify and analyze these responses according to individual vulnerability through the magnitude of the reduction of strength following eccentric exercise. Methods: Eighteen healthy subjects (11 men and seven women aged 18 to 71 years) performed five sets of six maximal isokinetic eccentric actions of the elbow flexors using an isokinetic dynamometer $\left(90^{\circ} \mathrm{s}^{-1}\right.$, range of motion $\left.80-20^{\circ}\right)$. Maximum voluntary concentric muscle contraction (CVM), DMIT and CK pre-, post (except CK), 24h, 48h, 72h and 96h were analyzed after the protocol. Individuals were classified as "low" (BR) and "high" (AR) responders at the peak of CVM decrease post-exercise using a $k$-means cluster analysis and compared between groups for CVM, DMIT and CK with independent t-test. Results: The comparison between $B R$ and $A R$ showed higher CVM decrease for $A R(A R:-30.7 \pm 3.3 \%, B R:-14.1 \pm 2.2 \%, p<0.001)$ and higher DMIT also for AR group ( $A R: 33.0 \pm 8.6 \mathrm{~mm} ; B R: 10.2 \pm 2.5 \mathrm{~mm}, p=0.033$ ) however, the peak values of $C K$ activity

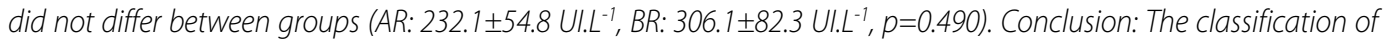
the subjects into low and high responders groups was important to demonstrate that the most responsive group to strength decrease after eccentric exercise also shows higher DMIT levels; notwithstanding, for creatine kinase this relation could not be established.

Keywords: exercise, muscle strength, skeletal muscle, resistance training.

\section{RESUMEN}

Introducción: El ejercicio excéntrico máximo promueve reducciones de capacidad de generación de energía, aumento de dolor muscular de aparición tardía (DMIT), y escape de creatina kinasa (CK) en la sangre. Sin embargo, estas respuestas al ejercicio excéntrico demostraron una gran variabilidad interindividual. Objetivos: Clasificar y analizar las respuestas de acuerdo a la vulnerabilidad individual al grado de disminución de la fuerza después del ejercicio excéntrico. Métodos: Dieciocho sujetos sanos (11 hombres y siete mujeres de entre 18 a 71 años) han realizado cinco series de seis acciones excéntricas isocinéticas máximas de los flexores del codo utilizando un dinamómetro isocinético $\left(90^{\circ} \mathrm{s}^{-1}\right.$, rango de movimiento 80-20\%). La contracción concéntrica muscular voluntaria máxima (CVM), 
DMIT y CK pre-, inmediatamente después (excepto CK), 24h, 48h, 72h y 96h fue analizada después del protocolo. Los individuos fueron clasificados en "bajos" (BR) y "altos" (AR) respondedores, conforme el pico de declinación en la CVM después del ejercicio mediante un análisis de cluster y comparados entre los grupos para la CVM, DMIT y CK con pruebas t-independientes. Resultados: La comparación entre BR y AR indicó mayor disminución de la CVM para $A R(A R:-30,7 \pm 3,3 \%, B R:-14,1 \pm 2,2 \%, p<0,001$ ) y mayor DMIT también para AR (AR: $33,0 \pm 8,6 \mathrm{~mm} ; B R: 10,2$ $\pm 2,5 \mathrm{~mm}, p=0,033$ ), sin embargo, los valores de pico de actividad de la CK no difirieron entre los grupos (AR:232,1 $\pm 54,8$ UI.L-1, BR: 306, $1 \pm 82,3$ UI.L-1, $p=0$ 0,490). Conclusión: La clasificación de los sujetos en los grupos bajos y altos respondedores fue importante para demostrar que el grupo más sensible a la disminución de la fuerza después del ejercicio excéntrico también muestra niveles más altos de DMIT. No obstante, para la creatina kinasa no se pudo establecer esta relación.

Palabras clave: ejercicio, fuerza muscular, músculo esquelético, entrenamiento de resistencia.

\section{INTRODUÇÃO}

O exercício excêntrico (EE) e os prejuízos funcionais ${ }^{1,2}$ e estruturais $^{3,4}$ advindos agudamente deste estímulo vem sendo amplamente estudados atualmente. Sobretudo quando o EE é realizado de maneira desacostumada, há rompimentos na ultraestrutura muscular com concomitantes reduções na capacidade de geração de força muscular e aumento da dor muscular percebida na musculatura exercitada, fenômenos estes que podem se estender por vários dias após o exercício $^{1,5,6}$. Adicionalmente, pode ocorrer aumento da permeabilidade de membrana sarcoplasmática, elevando a quantidade de proteínas intramusculares na corrente sanguínea ${ }^{5}$. A diminuição da força muscular, a sensação de dor e proteínas na corrente sanguínea são denominados marcadores indiretos de dano muscular e podem refletir a magnitude de dano muscular induzida pelo protocolo de EE. Entretanto, esses marcadores nem sempre apresentam respostas similares entre indivíduos desacostumados ao EE.

De fato, estas respostas podem apresentar grande variabilidade interindivíduos devido a fatores como grau de exposição prévia ao $\mathrm{EE}^{7}$, idade ${ }^{8}$ e o gênero ${ }^{9}$, dificultando o conhecimento real das respostas geradas pelo processo de dano muscular induzido pelo EE. Ainda que marcadores diretos de dano muscular (e.g., rompimentos de linha-Z) possam demonstrar uma resposta mais fidedigna quanto ao entendimento deste processo, eles indicam uma região muito específica da musculatura envolvida no EE, pois a análise é realizada apenas com uma pequena amostra de tecido muscular obtida por meio de biópsia muscular. Desta forma, uma análise das respostas individuais ao EE e, mais importante, a classificação dos sujeitos baseada na magnitude da redução da função contrátil muscular, agrupando-os em diferentes condições de resposta da capacidade de geração de força, pode auxiliar na redução da variabilidade e no entendimento das respostas ao EE6 .

A função contrátil do músculo, embora seja um marcador indireto de dano muscular, representa a atividade funcional da musculatura, o que num viés prático, juntamente com a dor muscular de início tardio (DMIT), se mostram como fatores importantes para o desempenho. Adicionalmente, foi sugerido que a utilização da avaliação da função muscular através da mensuração da força muscular seria interessante, pois reflete o estado geral do musculoesquelético ${ }^{6}$, se configurando o melhor marcador indireto de dano muscular ${ }^{10}$. Ainda que estudos prévios tenham indicado que os valores de $\mathrm{DMIT}^{11}$ e atividade de creatina quinase $(C K)^{12}$ pareçam não refletir tão acertadamente a magnitude do dano muscular, uma análise comparativa entre grupos de diferentes "respondedores" à queda de força após EE poderia ser interessante para melhor compreensão do processo de dano muscular.

Portanto, a proposta do presente estudo foi analisar a resposta da DMIT e o extravasamento de CK na corrente sanguínea de "baixos" e "altos" respondedores à queda da capacidade de geração de força após $E E$, independentemente de gênero ou idade. Hipotetisamos que os indivíduos com maior redução na força muscular após o EE, também apresentarão maiores níveis de DMIT e atividade de CK sanguínea.

\section{MÉTODOS}

Participaram do estudo 18 voluntários saudáveis (11 homens e sete mulheres com idade entre 18-71 anos (tabela 1). Todos os voluntários foram avaliados por um médico e considerados aptos para participar do estudo. Como critérios de inclusão nenhum dos sujeitos poderiam apresentar qualquer patologia cardíaca, diabetes mellitus e lesão musculoesquelética. Além disso, os voluntários deveriam estar ao menos 12 meses sem realizar treino de força, não poderiam fazer uso de nenhum medicamento anti-inflamatório ou suplemento alimentar. Os voluntários assinaram o termo de Consentimento Livre e Esclarecido que versa sobre os possíveis riscos e benefícios envolvidos com o estudo e o presente estudo foi aprovado pelo Comitê de Ética da Universidade Estadual de Campinas (Unicamp), Campinas, SP, Brasil.

Todos os voluntários foram avaliados três dias antes do início dos testes para reprodutibilidade da contração voluntária muscular concêntrica máxima (CVM). Subsequentemente, a CVM, dor muscular de início tardio (DMIT) e atividade de creatina quinase (CK) foram realizadas nos momentos imediatamente pré-, imediatamente pós- (exceto CK), 24h, 48h, 72h e 96h após o EE.

O protocolo de exercício excêntrico (EE) consistiu de cinco séries de seis ações excêntricas máximas de flexores de cotovelo à $90^{\circ} \mathrm{s}^{-1}$ por uma amplitude de movimento de $60^{\circ}\left(80-20^{\circ}\right)$ com 60 s de pausa entre séries, realizadas em dinamômetro isocinético (Biodex System Pro 4 , Nova lorque, EUA) ${ }^{1}$. O zero anatômico foi considerado como $0^{\circ}$. A articulação do ombro ficou à $90^{\circ}$ de flexão e $0^{\circ}$ de abdução, o antebraço se manteve supinado, segurando o braço de alavanca do dinamômetro

Tabela 1. Características antropométricas dos sujeitos, contração voluntária muscular concêntrica máxima (CVM) pré e trabalho total e a média de torque excêntrico (TE) desenvolvidos no exercício excêntrico (média \pm EPM).

\begin{tabular}{c|c|c|c|c|c|c}
\hline & $\begin{array}{c}\text { Idade } \\
\text { (anos) }\end{array}$ & $\begin{array}{c}\text { Massa } \\
\text { corporal } \\
(\mathbf{K g})\end{array}$ & $\begin{array}{c}\text { Altura } \\
(\mathbf{m})\end{array}$ & $\begin{array}{c}\text { CVM pré } \\
\mathbf{( N m})\end{array}$ & $\begin{array}{c}\text { Trabalho total } \\
(\mathbf{J})\end{array}$ & $\begin{array}{c}\text { TE } \\
\mathbf{( N m})\end{array}$ \\
\hline $\begin{array}{c}\text { Baixos } \\
\text { Respondedores } \\
(\mathrm{n}=10)\end{array}$ & $46,7 \pm 6,9$ & $78,7 \pm 3,7$ & $1,70 \pm 0,04$ & $36,71 \pm 3,44$ & $1070,77 \pm 139,12$ & $47,02 \pm 4,79$ \\
\hline $\begin{array}{c}\text { Altos } \\
\text { Respondedores } \\
(\mathrm{n}=8)\end{array}$ & $30,5 \pm 5,9$ & $66,2 \pm 5,6$ & $1,70 \pm 0,04$ & $36,94 \pm 6,26$ & $1114,59 \pm 211,31$ & $46,97 \pm 7,34$ \\
\hline
\end{tabular}


e os voluntários eram fixados no assento do dinamômetro por meio de faixas que passam na região do braço, tórax e coxa. Antes dos procedimentos no dinamômetro, foi realizado um primeiro aquecimento de duas séries de três repetições concêntricas/concêntricas à $90^{\circ} \mathrm{s}^{-1}$. Foi realizado um segundo de contração isométrica máxima na posição inicial antes de cada ação excêntrica e o membro retornava passivamente à posição inicial à $5^{\circ} \mathrm{s}^{-1}$. Todos os voluntários foram igualmente encorajados verbalmente a realizar o máximo de força possível em cada repetição e o trabalho total e o pico de torque excêntrico de cada série foram identificados usando o software do Biodex.

Para a análise da CVM todos os participantes foram posicionados no dinamômetro assim como explicado acima para o EE e realizaram uma série composta de quatro contrações máximas concêntricas à $60^{\circ} \mathrm{s}^{-1} \mathrm{com}$ a mesma amplitude de movimento do EE $\left(60^{\circ}, 20^{\circ}-80^{\circ}\right)$. O zero anatômico foi considerado como $0^{\circ}$. Todos os voluntários foram igualmente encorajados a realizar máxima força em todas as contrações e o pico de torque concêntrico para as quatro contrações foi considerado ${ }^{13}$. O coeficiente de variação da CVM calculado com duas medidas pré (separadas por três dias) foi de 4,3\% e o coeficiente de correlação intraclasse foi de 0,99.

A DMIT foi analisada utilizando uma escala visual analógica de 100 mm (0 mm: nenhuma dor, 100 mm: extremamente doloroso). Os voluntários flexionavam e estendiam ativamente o membro utilizado no protocolo de EE pela máxima amplitude possível e avaliaram separadamente a dor percebida assinalando uma marca na escala. Também foi avaliada a dor percebida após o investigador palpar a porção média do braço utilizado no EE. Os valores marcados na escala foram posteriormente medidos em milímetros. O mesmo investigador realizou todas as avaliações e a pressão de palpação foi mantida tão constante quanto possível. O valor pré da DMIT foi considerado como sendo zero e o valor médio das três avaliações (flexão, extensão e palpação) foi considerado'.

Para análise da atividade sérica de CK, foram coletados da veia antecubital aproximadamente $5 \mathrm{~mL}$ de sangue ${ }^{1}$. As amostras foram mantidas à temperatura ambiente por 10-15 min para coagular e, posteriormente, centrifugadas no local por $10 \mathrm{~min}$ para obter o soro que foi estocado em biofreezer $-70^{\circ}$ para análise posterior. As amostras foram analisadas em duplicata em um espectrofotômetro (Modelo U-5100, Hitachi, Tóquio, Japão) com um kit (CK-NAC, Laborlab, Guarulhos, Brasil). O coeficiente de variação intra- e inter-ensaio foram $6 \%$ e 4,5\%, respectivamente. 0 valor de referência de atividade de CK neste método é 24-190 UI.L-1

\section{Análise estatística}

Foi realizada a análise de agrupamento (K-means cluster) utilizando o software SPSS versão 19.0 (IBM, Nova lorque, EUA) para identificar os clusters resposta baseados no pico individual de queda da CVM depois de realizado o EE independentemente de gênero ou idade. Definimos dois clusters: baixos e altos respondedores à queda de CVM após o protocolo de EE. As características dos sujeitos, o trabalho total e a média de torque excêntrico desenvolvidos no EE, o pico de mudança de CVM, o pico de DMIT sentida e o pico da atividade sérica de CK foram comparados entre os clusters utilizando teste- $t$ independente. $O$ teste de Levene foi utilizado para avaliar a igualdade de variâncias em medidas independentes. Os dados estão apresentados em médias \pm erro padrão (EPM) salvo indicação diferente, e o nível de significância estatística estabelecido foi de 95\% $(p<0,05)$.

\section{RESULTADOS}

O procedimento de classificação estatística em clusters em dois grupos, baseado na queda da CVM após o EE, resultou em 10 sujeitos no grupo de baixos respondedores (BR) e oito sujeitos no grupo de alto respondedores (AR) (figura 1).

Foram observados valores similares entre as características antro-

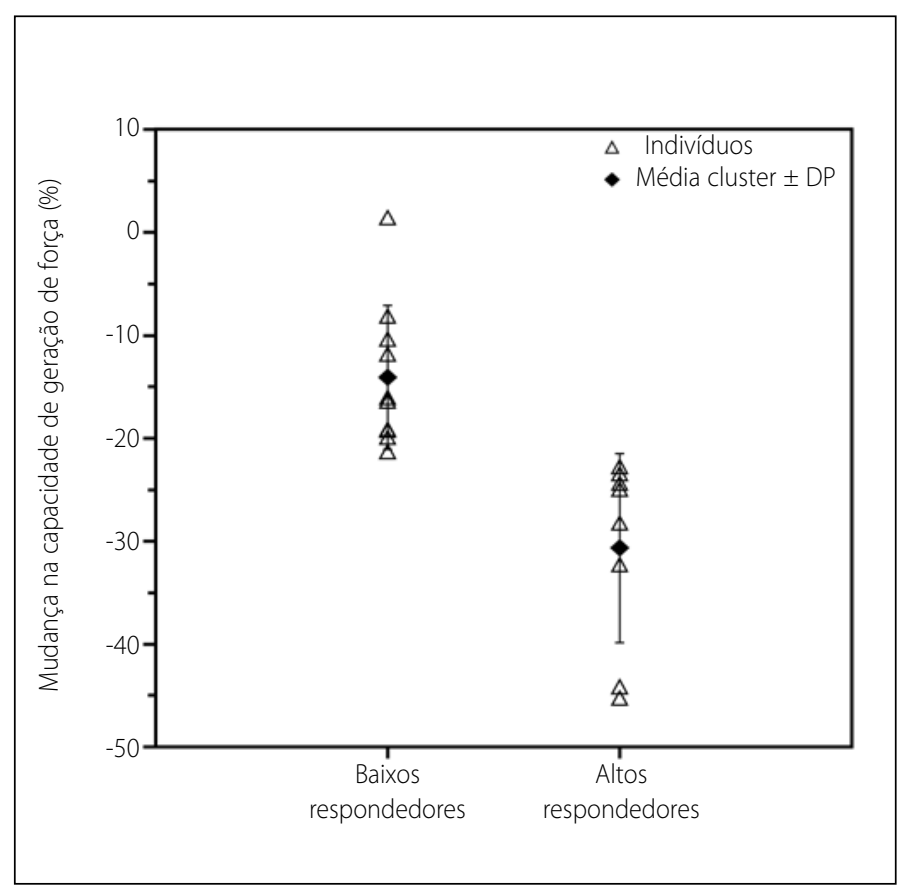

Figura 1. Classificação estatística em clusters de baixos e altos respondedores baseada no pico de mudança da força muscular após protocolo de exercício excêntrico máximo de flexores de cotovelo.

pométricas dos sujeitos entre clusters (idade, $p=0,101$; massa corporal, $p=0,074$; altura, $p=0,862$ ). Adicionalmente, os valores de CVM pré, o trabalho total e a média de torque excêntrico desenvolvidos no protocolo de $\mathrm{EE}$, foram similares entre clusters ( $p=0,974, p=0,860$ e $p=0,995$, respectivamente) (tabela 1).

Como esperado, a análise resultou em maior $(p<0,001)$ queda de CVM para o grupo AR comparado ao grupo BR (-30,7 $\pm 3,3 \%$ vs. $-14,1$ $\pm 2,2 \%$, respectivamente) após o EE (figura $2 \mathrm{~A}$ ).

Interessantemente o grupo que obteve maior redução nos valores de CVM após o EE, o AR, também relatou maiores valores de DMIT nos flexores do cotovelo (BR: 10,2 $\pm 2,5$ mm; AR: 33,0 $\pm 8,6$ mm, $p=0,033$ ) após o protocolo (figura 2B).

No entanto, este padrão não se estabeleceu para o pico de atividade de CK, uma vez que os grupos demonstraram extravasamento similar de CK (BR: 306,1 \pm 82,3 UI.L-1; AR: 232,1 $\pm 54,8$ UI.L-1 $, p=0,490$ ) após o EE (figura 2C).

\section{DISCUSSÃO}

O objetivo do presente estudo foi classificar e analisar as respostas da DMIT e CK em "baixos" e "altos" respondedores à queda da capacidade de geração de força após EE máximo de flexores do cotovelo, independentemente do gênero ou idade. Os principais resultados demonstraram que altos respondedores à queda de força obtiveram maiores níveis de DMIT após o protocolo, porém a resposta da CK não diferiu entre baixos e altos respondedores.

Estudos prévios questionam a relação entre a DMIT'11 e o extravasamento de CK com outros marcadores de dano muscular, indicando que estes marcadores não são bons preditores da magnitude de dano muscular induzido pelo exercício ${ }^{5,11,12,14}$. Um dos questionamentos em relação a utilização da CK como marcador indireto de dano muscular é pelo fato da molécula de CK possuir grande massa molecular (80 kDa) e, portanto, teria dificuldade em entrar no endotélio microvascular ${ }^{15}$. Desta forma, um pequeno grau de dano muscular que não altere significativamente a permeabilidade da membrana, não faria com que houvesse extravasamento de CK para a corrente sanguínea. O presente estudo, mesmo classificando os indivíduos em grupos baixos e altos respondedo- 
Baixos respondedores $\square$ Altos respondedores

A

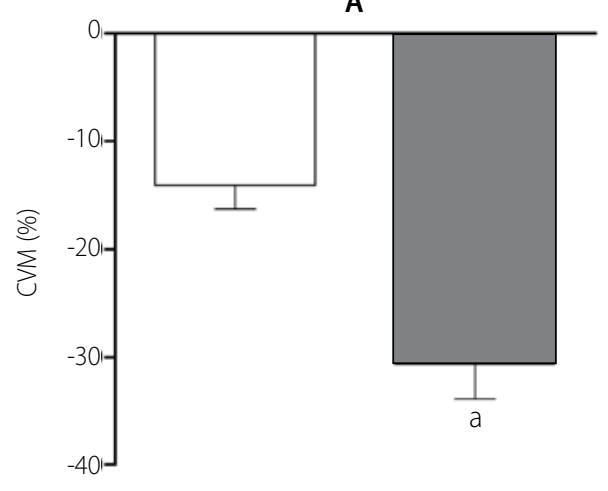

B

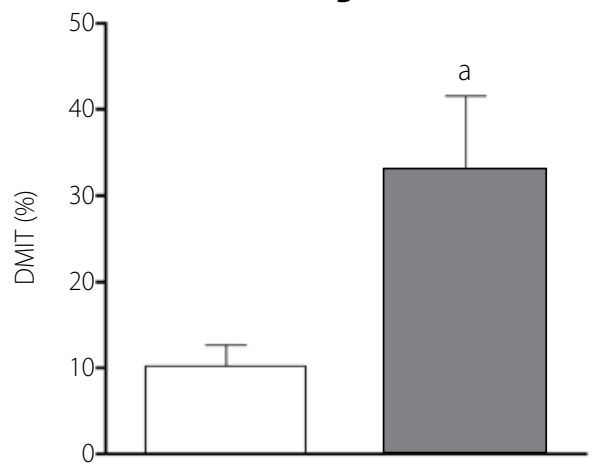

C

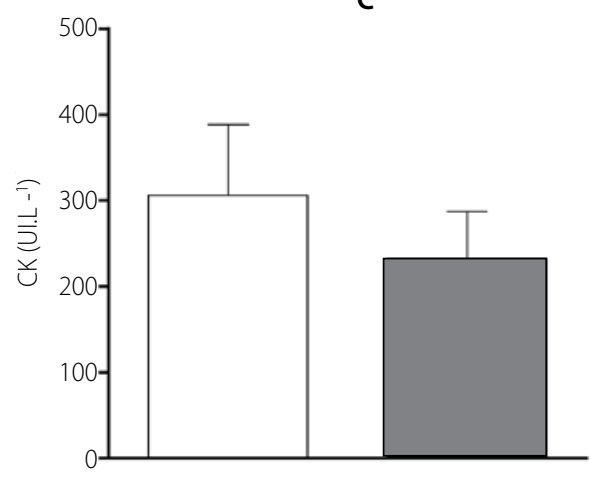

a Diferença significante para o grupo de baixos respondedores $(P<0,04)$.

Figura 2. Mudanças normalizadas (média \pm EPM) na contração voluntária máxima $(C V M$, pré-exercício $=0)(A)$; na dor muscular de início tardio $(\mathrm{DMIT}$, pré-exercício $=0)$ (B); e pico absoluto de atividade de creatina quinase (CK) sérica (C) após exercício excêntrico máximo de flexores do cotovelo em baixos e altos respondedores segundo divisão em cluster.

res à queda de CVM, não observou diferenças significantes na atividade sérica de CK corroborando com estudos que indicam que a análise do extravasamento de proteínas intramusculares na corrente sanguínea deve ser desencorajado para propostas de quantificação de prejuízos funcionais e/ou do grau de dano muscular induzido por EE ${ }^{12,14}$.

No que concerne a DMIT, Nosaka et al. ${ }^{11}$ demonstraram com uma amostra de 110 homens jovens que a dor muscular tardia possui correlações fracas ou mesmo inexistentes com outros marcadores indiretos de dano muscular (e.g. força muscular, amplitude de movimento, circunferência de membro e CK). Este resultado indica que não necessa- riamente os grupos que demonstraram maiores níveis de DMIT foram os grupos que indicaram maiores mudanças nos outros marcadores de dano muscular. No entanto, é importante notar que o estudo em questão não agrupou os indivíduos em clusters baseados nas respostas funcionais relacionadas com o dano muscular. Realizando esta análise, o presente estudo demonstrou que o grupo que se mostrou como sendo o mais vulnerável ao EE, com maior queda na capacidade de geração de força, foi também o grupo que relatou maiores níveis de DMIT. Portanto, assim como proposto anteriormente ${ }^{6}$, uma análise de clusters da resposta à queda de força foi importante para estabelecer uma relação entre a dor tardia induzida pelo protocolo e a vulnerabilidade à queda de força após o estímulo do EE.

O grupo de baixos respondedores demonstrou pico da queda de força de $\sim 14,1 \%$, enquanto que os altos respondedores obtiveram queda de força após o EE de $\sim 30,7 \%$. Interessantemente, essa diferença no déficit de força após o protocolo (aproximadamente 54\% maior para o grupo AR comparado ao grupo BR) não foi dependente dos níveis de contração voluntária máxima pré, do trabalho total ou torque excêntrico realizados no EE (sem diferenças entre os grupos), corroborando com sugestões prévias ${ }^{16}$. É preciso notar que o pico de queda da capacidade de geração de força que foi utilizado em nosso estudo para a divisão em clusters levou em consideração apenas os valores de 24h-96h após o protocolo, uma vez que a medida imediatamente após o EE pode ter grande interferência da fadiga induzida pelo protocolo e não somente efeito do dano muscular na capacidade de geração de força ${ }^{17}$.

O presente estudo também avaliou a resposta individual da capacidade de geração de força e classificou a magnitude de dano muscular sofrida pelos sujeitos de acordo com o proposto recentemente por Paulsen et al. ${ }^{6}$ em seu artigo de revisão. Desta forma, os sujeitos do grupo BR sofreram dano muscular "suave", pois obtiveram queda de CVM menor que, ou, por volta de, 20\%, durante as primeiras $24 \mathrm{~h}$ e recuperação rápida da CVM (figura 3). Por outro lado, os sujeitos do grupo de altos respondedores sofreram dano muscular com magnitude moderada, pois a CVM caiu por volta de 25-35\% (com dois indivíduos demonstrando queda de $\sim 45 \%$ 24h após o EE) e quatro dias depois nenhum dos indivíduos deste grupo ainda tinha recuperado totalmente a capacidade de geração de força (figura 3).

Adicionalmente, a comparação entre os valores de CVM 24h com a medida realizada imediatamente após o EE pode ser interessante, pois é um indicativo de presença de dano muscular secundário (queda adicional na capacidade de geração de força) ${ }^{18}$. Desta forma, se a CVM continua a apresentar queda $24 \mathrm{~h}$ comparada à CVM medida imediatamente após o EE, sugere-se que mecanismos envolvendo aumento da resposta inflamatória, principalmente decorrente da infiltração dos neutrófilos ${ }^{19}$ e espécies reativas de oxigênio no tecido potencializariam a magnitude do dano muscular ${ }^{20}$. A análise da resposta individual à queda da CVM após o EE (figura 3) indica que somente dois dos 10 indivíduos do grupo BR (20\% do grupo) apresentou queda da força $24 \mathrm{~h}$ comparada com a medida imediatamente após o EE, sendo que o individuo deste grupo que apresentou maior queda adicional da CVM foi de 9,2\% (pós vs. 24h). Já no grupo AR, seis dos oito sujeitos (75\% do grupo) apresentaram queda adicional $24 \mathrm{~h}$ após o protocolo, com alguns valores de CVM apresentando queda adicional de mais de 20\% (26,0\% e 23,6\% comparando a CVM pós vs. 24h). Uma limitação do presente estudo foi não avaliar nenhuma medida direta de dano secundário. No entanto, embora não se possa concluir que houve a ocorrência desse fenômeno no presente estudo, pode-se inferir que indivíduos que apresentam maior suscetibilidade à queda de força após EE e, portanto, maior suscetibilidade ao dano muscular induzido por EE, podem apresentar dano secundário. 


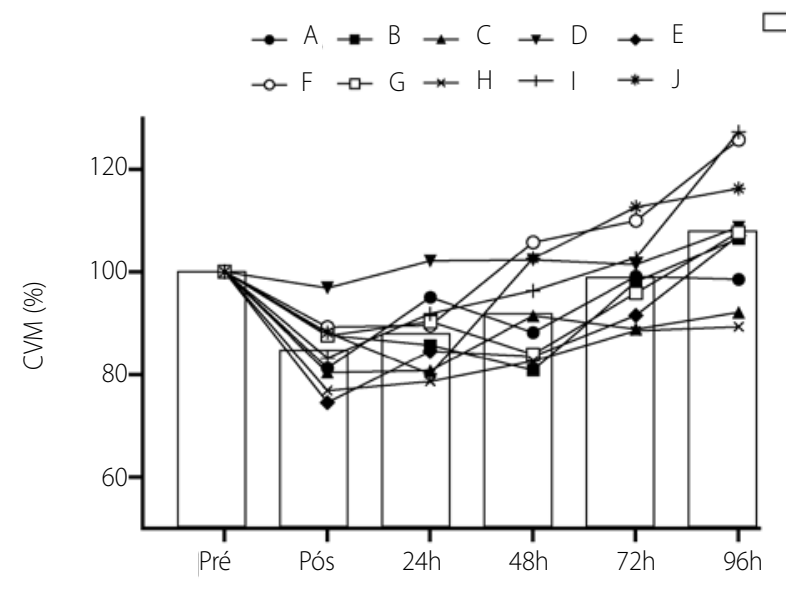

Médias $\rightarrow K \rightarrow L \rightarrow M \rightarrow N$

$\rightarrow O \rightarrow P \rightarrow Q \rightarrow R$

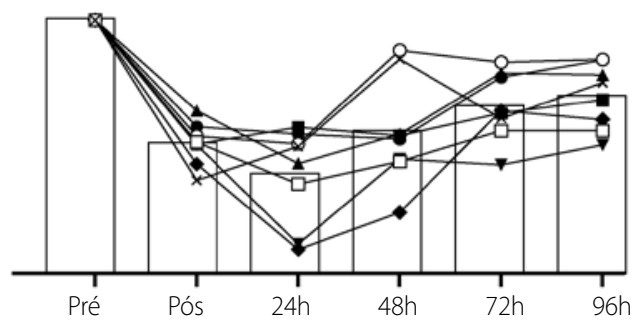

Tempo

Figura 3. Mudanças normalizadas individuais (cada letra representa um indivíduo) e médias da contração voluntária máxima (CVM, pré-exercício = 0) após exercício excêntrico máximo de flexores do cotovelo em baixos (esquerda) e altos (direita) respondedores segundo divisão em cluster.

\section{CONCLUSÃO}

Conclui-se que a classificação dos indivíduos segundo a mudança na capacidade de geração de torque foi importante para demonstrar que o grupo mais responsivo a queda de força após EE também evidencia maiores níveis de DMIT, no entanto, para a CK esta relação não pôde ser estabelecida. A classificação em clusters, separando indivíduos baseados na resposta da força muscular após o exercício, é uma boa maneira de analisar respostas geradas pelo dano muscular induzido por EE, diminuindo a variabilidade e possibilitando maior entendimento deste processo.

\section{AGRADECIMENTOS}

Agradecemos à Coordenação de Aperfeiçoamento de Pessoal de Nível Superior (CAPES) e ao Fundo de apoio ao Ensino, à Pesquisa e à Extensão (FAEPEX) da UNICAMP pelos auxílios concedidos.

Todos os autores declararam não haver qualquer potencial conflito de interesses referente a este artigo.

\section{REFERÊNCIAS}

1. Nogueira FR, Libardi CA, Nosaka K, Vechin FC, Cavaglieri CR, Chacon-Mikahil MP. Comparison in responses to maximal eccentric exercise between elbow flexors and knee extensors of older adults. J Sci Med Sport. 2014;17(1):91-5.

2. Conceição MS, Libardi CA, Nogueira FR, Bonganha V, Gáspari AF, Chacon-Mikahil MP, et al. Effects of eccentric exercise on systemic concentrations of pro- and anti-inflammatory cytokines and prostaglandin (E2): comparison between young and postmenopausal women. Eur J Appl Physiol. 2012;112(9):3205-13.

3. Yu JG, Carlsson L, Thornell LE. Evidence for myofibril remodeling as opposed to myofibril damage in human muscles with DOMS: an ultrastructural and immunoelectron microscopic study. Histochem Cell Biol. 2004;121(3):219-27.

4. Shepstone TN, Tang JE, Dallaire S, Schuenke MD, Staron RS, Phillips SM. Short-term high- vs. low-velocity isokinetic lengthening training results in greater hypertrophy of the elbow flexors in young men. J Appl Physiol (1985).2005;98(5):1768-76.

5. Clarkson PM, Hubal MJ. Exercise-induced muscle damage in humans. Am J Phys Med Rehabil. 2002;81(Suppl 11):S52-69

6. Paulsen G, Mikkelsen UR, Raastad T, Peake JM. Leucocytes, cytokines and satellite cells: what role do they play in muscle damage and regeneration following eccentric exercise? Exerc Immunol Rev. 2012;18:42-97.

7. Barroso R, Roschel H, Gil S, Ugrinowistch C, Tricoli V. Effect of the number and the intensity of eccentric muscle actions on muscle damage markers. Rev Bras Med Esporte. 2011;17(6):401-4.

8. Lavender AP, Nosaka K. Comparison between old and young men for changes in makers of muscle damage following voluntary eccentric exercise of the elbow flexors. Appl Physiol Nutr Metab. 2006;31(3):218-25.

9. Sayers SP, Clarkson PM. Force recovery after eccentric exercise in males and females. Eur J Appl Physiol. 2001;84(1-2):122-6.

10. Raastad T, Owe SG, Paulsen G, Enns D, Overgaard K, Crameri R, et al. Changes in calpain activity, muscle structure, and function after eccentric exercise. Med Sci Sports Exerc. 2010;42(1):86-95
11. Nosaka K, Newton M, Sacco P. Delayed-onset muscle soreness does not reflect the magnitude of eccentric exercise-induced muscle damage. Scand J Med Sci Sports. 2002;12(6):337-46.

12. Warren GL, Lowe DA, Armstrong RB. Measurement tools used in the study of eccentric contraction-induced injury. Sports Med. 1999;27(1):43-59.

13. Nogueira $F R$, Libardi CA, Vechin $F C$, Lixandrão $M E$, de Barros Berton RP, de Souza TM, Conceição MS, Cavaglieri CR, Chacon-Mikahil MP. Comparison of maximal muscle strength of elbow flexors and knee extensors between younger and older men with the same level of daily activity. Clin Interv Aging. 2013;8:401-7.

14. Manfredi TG, Fielding RA, O'Reilly KP, Meredith CN, Lee HY, Evans WJ. Plasma creatine kinase activity and exercise-induced muscle damage in older men. Med Sci Sports Exerc. 1991;23(9):1028-34.

15. Baty JJ, Hwang H, Ding Z, Bernard JR, Wang B, Kwon B, Ivy JL. The effect of a carbohydrate and protein supplement on resistance exercise performance, hormonal response, and muscle damage. J Strength Cond Res. 2007;21(2):321-9.

16. Chapman DW, Newton MJ, Zainuddin Z, Sacco P, Nosaka K. Work and peak torque during eccentric exercise do not predict changes in markers of muscle damage. Br J Sports Med. 2008;42(7):585-91

17. Nosaka K, Chapman D, Newton M, Sacco P. Is isometric strength loss immediately after eccentric exercise related to changes in indirect markers of muscle damage? Appl Physiol Nutr Metab. 2006;31(3):313-9.

18. Faulkner JA, Brooks SV, Opiteck JA. Injury to skeletal muscle fibers during contractions: conditions of occurrence and prevention. Phys Ther. 1993;73(12):911-21.

19. Kyriakides C, Austen W Jr, Wang Y, Favuzza J, Kobzik L, Moore FD Jr, et al. Skeletal muscle reperfusion injury is mediated by neutrophils and the complement membrane attack complex. Am J Physiol. 1999;277(6 Pt 1):C1263-8.

20. Smith C, Kruger MJ, Smith RM, Myburgh KH. The inflammatory response to skeletal muscle injury: illuminating complexities. Sports Med. 2008;38(11):947-69. 